\title{
Two-Dimensional Foam Rheology with Viscous Drag
}

\author{
E. Janiaud, ${ }^{*}$ D. Weaire, and S. Hutzler \\ School of Physics, Trinity College Dublin, Ireland \\ (Received 1 February 2006; published 20 July 2006)
}

\begin{abstract}
We formulate and apply a continuum model that incorporates elasticity, yield stress, plasticity, and viscous drag. It is motivated by the two-dimensional foam rheology experiments of Debregeas et al. [Phys. Rev. Lett. 87, 178305 (2001)] and Wang et al. [Phys. Rev. E 73, 031401 (2006)], and is successful in exhibiting their principal features, which are an exponentially decaying velocity profile and strain localization. Transient effects are also identified.
\end{abstract}

DOI: $10.1103 /$ PhysRevLett.97.038302

PACS numbers: 83.80.Iz, 82.70.Rr, 83.10.Ff

While initially two-dimensional (2D) foams were introduced only as a simple model system for numerical and theoretical studies [1,2], recent years have also seen a variety of rheological experiments on so-called quasi-2D foams, i.e., foams consisting of a single layer of bubbles [3-8]. Using bubbles trapped between two glass plates (Hele-Shaw cell) in a cylindrical Couette geometry (the foam is contained between two concentric cylinders), Debrégeas et al. found that the flow of the foam localizes near the inner moving wall with an exponential velocity profile, forming shear bands [4]. While quasistatic cellular simulations $[9,10]$ showed some agreement with the results, they continue to excite debate [7], especially in regard to the localization of shear and deformation [6], which is the salient feature of the experiment. Recently, Wang et al. have extended shear experiments to the simpler planar geometry [8]. While their experiments using bubbles between a liquid pool and a glass plate showed the formation of shear bands with an exponential velocity profile, a nearly linear velocity profile was obtained for a bubble floating on the liquid (bubble raft or Bragg raft). This has evidenced the crucial role played by the method used to confine the bubbles and indicates that the nonuniform stress imposed by the Couette geometry is not sufficient to explain the formation of shear bands with exponential decaying velocity.

In this Letter, we introduce an elementary continuum model for the analysis of rheological properties of a twodimensional foam. It includes a viscous drag that has no counterpart in conventional 3D foam rheology. Our model is therefore closely related to the $2 D$ viscous froth model [11] which was designed to enable dynamic simulations to be undertaken with the full cellular structure of the foam and included just such a viscous drag. Here the viscous drag will enter as a term in the continuum description, depending on a local average of the boundary velocity. Experiment and theory have already addressed this force as it arises in the flow of bubbles in cylindrical tubes and in narrow channels [5]. It is often associated with the name of Bretherton, who showed that the force varies with twothirds the power of velocity [12]. In some circumstances, a power law of one-half is suggested [13]. Nevertheless, as in the case of the 2D viscous froth, we adopt a linear form in order to keep the model and the analysis simple, in a search for a qualitative and semiquantitative understanding. In other respects, the model is akin to the familiar Bingham model of a substance that has a yield stress [14] and an internal viscosity. This, or one of its variants, is often invoked in the analysis of bulk foams. However, as in the recent work of Takeshi and Sekimoto [15], we also include an elastic response, so that the model we propose has four key ingredients: elasticity up to a yield stress, plasticity, internal viscosity, and a viscous drag force.

While it is amenable to obvious generalization, the model will be defined here for the simple planar shear geometry, as in [8]. Displacement $u(y, t)$ and the velocity $v(y, t)=\frac{\partial u(y, t)}{\partial t}$ are in the $x$ direction only, as when shear takes place between two parallel infinite boundaries in that direction (see Fig. 1). This reduces the problem to one dimension. Strain and strain rate are reduced to scalars $\gamma(y, t)=\frac{\partial u}{\partial y}$ and $\dot{\gamma}(y, t)=\frac{\partial \gamma}{\partial t}$.

We will neglect inertia throughout, so that the total force acting on an element of fluid at $y$ must be zero. Forces arise from the gradient of the shear stress $\sigma(y, t)$ and the drag

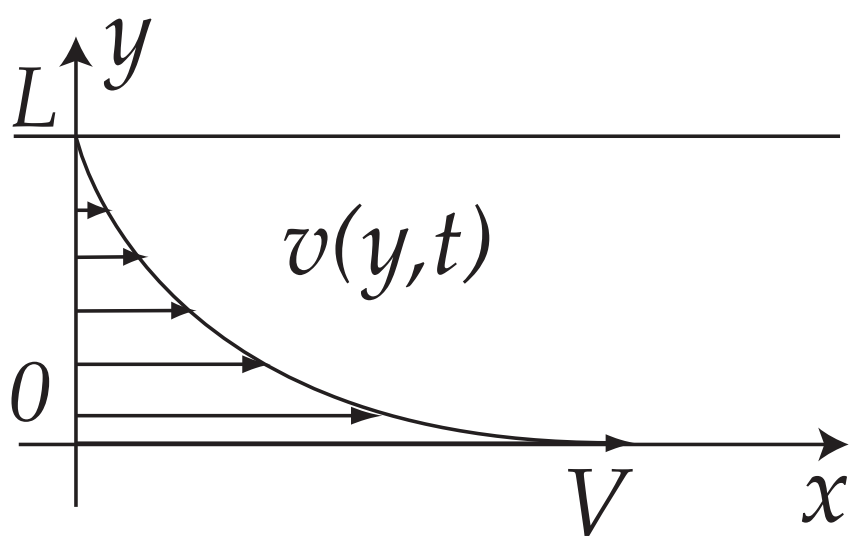

FIG. 1. Geometry of the case considered here in which the velocity $v(y, t)$ and the displacement $u(y, t)$ are functions of the $y$ coordinate and time $t$. 
force per unit area, $F=-\beta v$, where $\beta$ is the mean drag coefficient. In two dimensions, stress has the dimension of a force divided by a length and $\beta$ is expressed in units of force $\times$ time per volume. The required force balance is

$$
\frac{\partial \sigma}{\partial y}=\beta v
$$

It remains to specify the constitutive relation for $\sigma$ in terms of $\gamma$ and $\dot{\gamma}$. For simplicity, we capture the desired ingredients of elasticity, yield stress, and plasticity with the following relation:

$$
\sigma=\sigma_{Y} f\left(\gamma / \gamma_{Y}\right)+\eta \dot{\gamma}
$$

Here, $\sigma_{Y}$ is the yield stress and $\gamma_{Y}$ is the yield strain. We choose $f\left(\gamma / \gamma_{Y}\right)=\tanh \left(\gamma / \gamma_{Y}\right)$, which roughly corresponds to a typical 2D static stress-strain relation for foams [14]. For foams $\gamma_{Y}$ is of the order of unity and we shall set it equal to unity here. The final term in Eq. (2) is the usual strain-rate term of the Bingham model. Note that for foams the viscosity $\eta$ depends on the strain $\gamma$. For low strain, the dissipation is due to the stretching of films and occurs at the same rate as the applied deformation. For high strain, it is mainly due to topological changes which lead to the disappearance and creation of films. This occurs at much higher rate than the applied deformation [16]. Nevertheless, the assumption of a constant viscosity is helpful in our elementary model. A very important restriction requires that Eq. (2) is used only when the strain rate $\dot{\gamma}$ always has the same sign (negative in what follows), which is the case in the experiments to which we are referring. In further work we will include hysteretic effects, which are very important, but for now we accept this restriction.

We can nondimensionalize Eqs. (1) and (2) by introducing the natural length scale $L_{0}=(\eta / \beta)^{1 / 2}$ and natural time scale $T_{0}=\eta / \sigma_{Y}$. From now, length and time will be expressed in units of $L_{0}$ and $T_{0}$. A convenient representation of Eqs. (1) and (2) is

$$
\frac{\partial^{2} v}{\partial y^{2}}-v=-\frac{\partial}{\partial y} f\left(\frac{\partial u}{\partial y}\right)
$$

where

$$
v=\frac{\partial u}{\partial t} .
$$

The model can be solved analytically in various cases and limits. More generally, a numerical scheme of integration can be used to follow the time dependence of the variables, as follows. We discretise $y$ and $t$ with small steps $\Delta y$ and $\Delta t$, using lowest order expressions for derivatives. Given a knowledge of $u$ in steps up to time $t, \frac{\partial u}{\partial t}$ may be estimated as a backward derivative and Eq. (3) may be solved for $v(y, t)$ with the imposed boundary conditions. Equation (4) then enables us to update $u$ to $t+\Delta t$. (In practice, an improved Euler method was used for the integration in time.)
We will consider only the case in which the boundary at $y=0$ is given a finite velocity $V$ at all times $t$, while the boundary at $y=L$ is held fixed. Correspondingly, $u(y=$ $0, t)=V t$ and $u(y=L, t)=0$. For the results presented here, we set $L=15$ and $V$ takes various values. The quantity $\Gamma=V t / L$ may be regarded as the total applied shear at time $t$.

The numerical results presented in Fig. 2 are for low velocities $V \ll 1$ and show the existence of several regimes as the total applied shear is increased.

Regime I is observed for small total applied shear, at which both velocity and strain profiles [Figs. 2(a) and 2(b)] are close to exponentials. Regime II is characterized by a linear velocity profile and a homogeneous strain. In regime III both velocity and strain profiles combine an exponential decay close to the moving boundary and a linear decay close to the fixed boundary. With further increase of total applied shear the linear tails diminish, leading to an asymptotic steady state (regime IV) similar to that for small applied shear.

The existence of these distinct regimes is also evident from the plots of strain rates and stress as a function of total applied shear as shown in Figs. 2(c) and 2(d), respectively. While regime I is characterized by a strong localization of both strain rate and stress, in regime II $\left(10^{-2}<\Gamma<1\right)$ the strain rate is homogeneous. The asymptotic steady state of regime IV is again characterized by strong localization of the strain rate. Stress is saturating to its maximal magnitude 1 for all values of $y$.
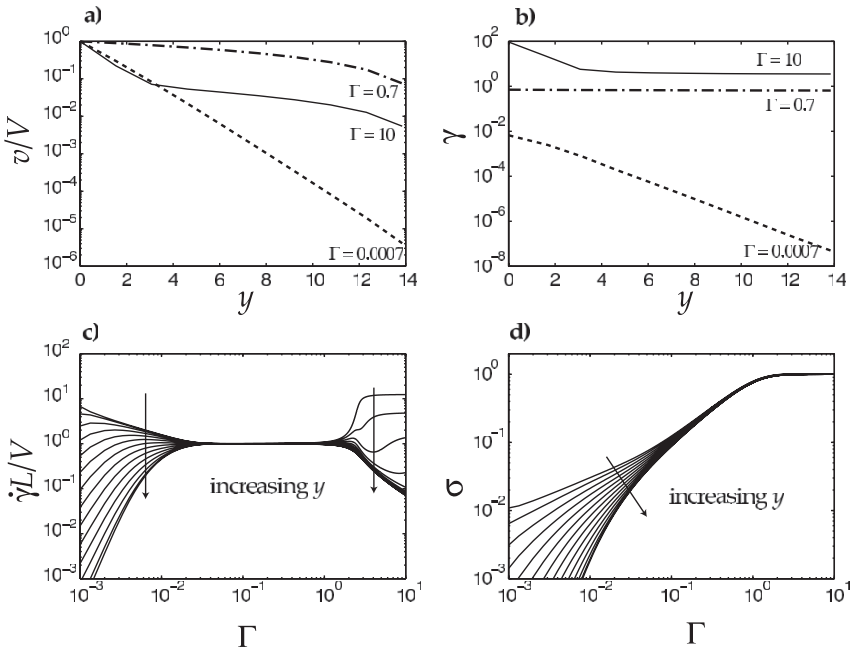

FIG. 2. (a),(b) Profiles of velocity $v / V$ and magnitude of the strain $\gamma$ for three different times, represented by the total applied shear $\Gamma=V t / L$, shown in semilog scale. This exemplifies three regimes of exponential or linear profiles. The regimes also feature (c) the variation of the magnitude of strain rate $\dot{\gamma}$ and (d) the variation of the magnitude of stress $\sigma$ with total applied shear $\Gamma$, shown in log-log scale. In all the calculations shown, we have chosen $L=15$ and a low boundary velocity of $V=0.005$ at $y=0$. 

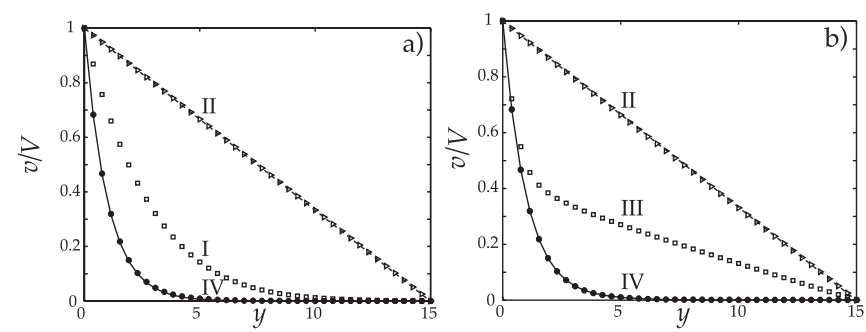

FIG. 3. Velocity profiles scaled by $V$ for $L=15$ [numerical results for $V=0.001(\triangleright), V=0.3(\square)$, and $V=60(\bullet)]$. (a) is for total applied shear $\Gamma=0.1$ and shows the succession of regime II, regime I, and finally regime IV. (b) corresponds to the transition from regime II to III to IV as obtained for $\Gamma=1$. The dashed line represents the linear solution given by Eq. (8), and the solid line is the steady states solution given by Eq. (6) corresponding to the exponential localization.

Figure 3 shows the velocity profiles obtained for the same total applied shear but for different shearing velocity $V$. For $V \ll 1$, the velocity varies linearly, corresponding to regime II. For $V \gg 1$, the profile approaches the asymptotic form (regime IV). For $V \approx 1$, we can have either regime I [for $\Gamma=0.1$ on Fig. 3(a)] or regime III [for $\Gamma=$ 1 on Fig. 3(b)] where we see an initial exponential decay followed by a linear tail (regime III). Figure 4 represents the different regimes encountered on a semiquantitative $\Gamma-V$ diagram. Depending on the shearing velocity $V$, several scenarios are possible before reaching the steady state of the regime IV.

In order to understand these features, we return to the governing Eq. (3) and reduce it by various approximations. For small time (regime I), $u$ is small and we neglect the right-hand side of Eq. (3), which is approximately $-\frac{\partial^{2} u}{\partial y^{2}}$. The remaining equation,

$$
\frac{\partial^{2} v}{\partial y^{2}}-v=0
$$

has the elementary solution

$$
v=V \frac{\sinh (L-y)}{\sinh (L)}
$$

Note that this solution does not vary with time, implying that the system jumps instantaneously to the above velocity profile. This is indeed consistent with what is found in the numerical treatment and is a consequence of the singular initial condition and the neglect of inertia. Provided $L \gg 1$ in the reduced units, this solution is approximately an exponential over most of the range. The exponential profile survives until $\frac{\partial^{2} u}{\partial y^{2}}$ becomes large and overtakes the term proportional to $v$.

Neglecting the term proportional to $v$ in Eq. (3), rather than that on the right-hand side, and approximating the latter as already stated, we obtain

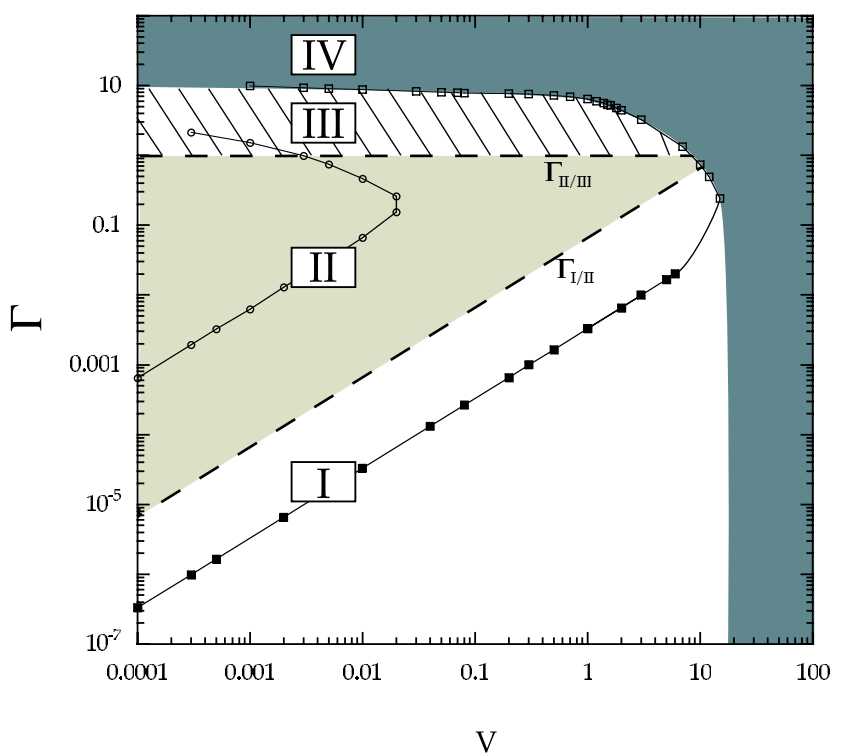

FIG. 4 (color online). Qualitatively different velocity profiles are found in different regions of the $\Gamma-V$ diagram. Regime I: exponential. Regime II: linear. Regime III: combined exponential/linear. Region IV: approach to final steady state velocity profile with an exponential localization. The boundaries $\Gamma_{\mathrm{I} / \mathrm{II}}$ and $\Gamma_{\text {II } / \text { III }}$ (dashed lines) are defined as in the text. The area to the left of the computed $\bigcirc$ data points is defined so that the relative error between the velocity profile and the linear profile characterizing regime II [Eq. (8)] is smaller than 1\%. A similar 1\% threshold based on Eq. (6) has been used as a numerical criterion for the computation of the boundary between regimes III and IV ( $\square$ ). This threshold is also used for the computation of the $\mathbf{a}$ data points.

$$
\frac{\partial^{2} v}{\partial y^{2}}=-\frac{\partial^{2} u}{\partial y^{2}} .
$$

Hence $u+v=a(t) y+b(t)$. Writing $v=\frac{\partial u}{\partial t}$ and integrating again gives $u=A(y) e^{-t}+a(t) y+b(t)$. This shows that the solution which develops after some time is linear, with a decaying transient part. The decay time is unity, in the units used. Applying the boundary conditions at $y=0$ and $y=L$, the linear variation of the velocity is then given by

$$
v=V\left(1-\frac{y}{L}\right)
$$

in excellent agreement with the simulation (see Fig. 3).

A further transition (regime III) takes place when the approximation $\tanh z \sim z$ fails, and can be replaced by $\tanh \sim 1$, as the strain $\gamma$ increases beyond the yield strain $\gamma_{Y}$. At any given time in this regime, the second approximation replaces the first for $y>y_{0}$. Thus the same exponential of regime I is to be expected for $y<y_{0}$, continued by the linear solution of regime II for $y>y_{0}$.

As the time $t$ tends to infinity (regime IV), $y_{0}$ tends to $L$ and the solution returns to the effectively exponential form 
of Eq. (6). The simulations are in excellent agreement with this profile, as shown by the solid line in Fig. 3. This solution is only asymptotically reached. A closer analysis of this approach is possible but will not be pursued here: suffice it to say that it is a slow (power law) convergence.

The boundaries between these regions may be identified as follows. That between regimes I and II may be found by estimating and equating the magnitudes of the terms neglected in their respective approximations. Using the solutions given in Eqs. (6) and (8) gives $\Gamma_{\mathrm{I} / \mathrm{II}} \sim V / L$, in agreement with the linearity in $V$ found in numerical computation of the I/II boundary (see Fig. 4). Similarly, we enter regime III when the maximum value of strain $\gamma$ reaches $\gamma_{Y}$, which for the linear solution occurs at $\Gamma_{\text {II } / \mathrm{III}} \sim$ 1 , which is in reasonable agreement with the numerical data shown in Fig. 4. Putting these together, we see that regime II is eliminated entirely for $V>L$ in dimensionless units. Reinstalling physical units, this corresponds to a shear rate which exceeds $\sigma_{y} / \eta$.

The steady state obtained at high applied shear $\Gamma$ offers a very elementary candidate for the explanation of the phenomenon of localization with exponential velocity profiles in 2D foams $[4,8]$. Our results allow for a direct comparison with the planar shear experiment on 2D foams of Ref. [8]. As Eq. (6) can be approximated (in physical units) by $v / V \approx \exp \left(-y / L_{0}\right)$, the velocity measurements provide us with a direct determination of $L_{0}=(\eta / \beta)^{1 / 2}$. Expressed in units of bubble diameter $d$, they correspond to $L_{0} \approx d$ for the bubbles trapped between a glass plate and a pool of liquid. Our model also explains why no exponential localization is found in the experiments using bubble rafts. In this case, the mean drag coefficient $\beta$ is expected to be very small, since there are no rigid plates, but rather the foam slides on underlying liquid. The decay length of the exponential which scales like $(\eta / \beta)^{1 / 2}$ increases up to a value of the same order of magnitude as the system size $L$ and the velocity profile appears to be very close to a linear form. From Eq. (6) we also see, consistent with the experiments of [8], that in the steady state regime the scaled velocity profiles $v / V$ do not depend on the shear rate $V / L$.

Clearly the model can be applied more generally, for example, to the circular Couette geometry. This suggests the use of polar coordinates $(r, \theta)$, which leads to an extra term $\sigma / r$ in the divergence of the stress of Eq. (1). Although a full mathematical treatment is required to solve the problem in the general case, it is possible to use our present results, provided this extra term is much smaller than the viscous drag term $\beta v$. Assuming that the stress is dominated by the viscous contribution $\eta \dot{\gamma}$ during the steady state, one finds that Eq. (1) still holds if the distance between the two cylinders is much bigger than $L_{0}$. This is the case when bubbles are confined in a Hele-Shaw cell where the velocity profiles are found to be exponential with $d<L_{0}<2 \mathrm{D}$ [4]. On the contrary, for a bubble raft sheared between two concentric cylinders, the velocity profile is not exponential but rather discontinuous [7]. This can be explained by a viscous drag too small to overcome the nonuniform stress effect of the Couette geometry.

We thus have seen that the exponentially decaying velocity profile in 2D foams, which is the signature of shear bands, is due the viscous drag generated by the bubbles on the confining plate. In due course, more realistic forces (e.g., the Bretherton form) may be required, at the expense of the extreme simplicity of what we have shown here. Most of the qualitative conclusions are likely to remain intact.

Our work was supported by the European Space Agency (No. MAP AO-99-108:C14914/02/NL/SH and No. MAP AO-99-075:C14308/00/NL/SH) and Science Foundation Ireland (No. RFP 05/RFP/PHY0016).

*Electronic address: janiaude@tcd.ie

[1] F. Bolton and D. Weaire, Phys. Rev. Lett. 65, 3449 (1990).

[2] D. J. Durian, Phys. Rev. E 55, 1739 (1997).

[3] M. F. Vaz and S. J. Cox, Philos. Mag. Lett. 85, 415 (2005).

[4] G. Debrégeas, H. Tabuteau, and J.-M. di Meglio, Phys. Rev. Lett. 87, 178305 (2001).

[5] I. Cantat, N. Kern, and R. Delannay, Europhys. Lett. 65, 726 (2004).

[6] E. Janiaud and F. Graner, J. Fluid Mech. 532, 243 (2005).

[7] J. Lauridsen, G. Chanan, and M. Dennin, Phys. Rev. Lett. 93, 018303 (2004).

[8] Y. Wang, K. Krishan, and M. Dennin, Phys. Rev. E 73, 031401 (2006).

[9] A. Kabla and G. Debrégeas, Phys. Rev. Lett. 90, 258303 (2003).

[10] S. J. Cox, Colloids Surf. A 263, 81 (2005).

[11] N. Kern, D. Weaire, A. Martin, S. Hutzler, and S. J. Cox, Phys. Rev. E 70, 041411 (2004).

[12] F. P. Bretherton, J. Fluid Mech. 10, 166 (1961).

[13] N. D. Denkov, V. Subramanian, D. Gurovich, and A. Lips, Colloids Surf. A 263, 129 (2005).

[14] D. Weaire and S. Hutzler, The Physics of Foams (Oxford University Press, Oxford, 1999).

[15] O. Takeshi and K. Sekimoto, Phys. Rev. Lett. 95, 108301 (2005).

[16] S. Hutzler, D. Weaire, S. J. Cox, A. van der Net, and E. Janiaud (to be published). 